EPSC Abstracts

Vol.14, EPSC2020-391, 2020, updated on 15 Aug 2020

Europlanet Science Congress 2020

(C) Author(s) 2020. This work is distributed under

the Creative Commons Attribution 4.0 License.

\title{
Linear polarization as a tool to characterize interplanetary, cometary, and extrasolar dust particles
}

Anny-Chantal Levasseur-Regourd ${ }^{1}$, Clément Baruteau ${ }^{2}$, Jérémie Lasue ${ }^{2}$, Julien Milli ${ }^{3}$, and JeanBaptiste Renard ${ }^{4}$

${ }^{1}$ LATMOS, Sorbonne Université, CNRS, CNES, Paris, France (aclr@latmos.ipsl.fr)

${ }^{2}$ IRAP, Université de Toulouse, CNES, CNRS, UPS, Toulouse, France

${ }^{3}$ IPAG, Université de Grenoble-Alpes, Grenoble, France

${ }^{4} \mathrm{LPC} 2 \mathrm{E}$, Université d'Orléans, CNRS, Orléans, France

\section{Summary}

Linear polarization observations have suggested the presence of dust particles that scatter solar light within cometary comae and the interplanetary dust cloud. Recent progresses result from in-situ observations or remote observations from large telescopes, while avoiding contamination from atmospheric airglow. The interpretation of polarimetric observations, at given phase angles and wavelengths, is a powerful tool to better understand the composition and physical properties of these irregular dust particles and their formation processes. It now provides clues to the origin of the Solar System and to some characteristics of dust in stellar systems, as discussed in [1].

\section{- Zodiacal light polarization}

Along a line-of-sight within the interplanetary dust cloud, both solar distance (R) and phase angle (a) vary, meaning that the intensity and linear polarization (P) of solar light scattered by interplanetary dust (i.e. zodiacal light) need to be inverted to retrieve some local properties. In the near-ecliptic symmetry plane of the cloud, $\mathrm{P}(\mathrm{a})$ phase curves are smooth, with a slightly negative branch below $20^{\circ}$ and a maximum by $90^{\circ}$. For a constant $a=90^{\circ}, \mathrm{P}$ increases with $\mathrm{R}$, at least between 0.5 au and 1.5 au. Numerical and experimental simulations (in the laboratory and under microgravity conditions) of such results suggest i) the presence in dust of low-absorbing minerals and absorbing complex organics, with a significant amount of fluffy aggregates, ii) some sublimation of semi-volatile organics with decreasing $\mathrm{R}$ and increasing temperatures [2; also 3-5 for reviews].

\section{- Cometary dust polarization}

In situ observations have, up to now, only been done on board Giotto during its flybys, allowing us to suggest the presence of a fragment in the coma of 26P/Grigg-Skjellerup and to derive very low values for geometric albedos (about $3 \%$ ) and densities ( 50 to $500 \mathrm{~kg} \mathrm{~m}^{-3}$ ) of $1 \mathrm{P} /$ Halley dust particles [6-7].

Dust whole-coma remote observations, which avoid contamination from gaseous emissions with narrow interference filters, have been used by various teams to obtain $P(a)$ phase curves. As for the zodiacal light, they are smooth; also P (whenever positive) increases with wavelenght for a given a. 
Numerical and experimental simulations are consistent with a dust population of irregular and possibly fractal structure, with complex optical indices suggesting mixtures of minerals and more absorbing organics [8-10]. Polarimetric in situ observations and remote imaging of comae (at given phase and wavelenght) suggest that the properties of dust are quite different in dust jets than elsewhere in a dust coma, and in Oort-cloud comets than in periodic comets [9, 11-12]. Such trends fairly agree with the ground-truth provided by the Rosetta rendezvous with 67P/ChuryumovGerasimenko [13 for a review]. Finally, experimental simulations of 67P pre-perihelion brightness phase curves provide clues to an important amount of organic compounds and to fluffy aggregates with sizes above 10 micrometres [14].

\section{- Significance of the results, back in time and beyond in space}

Back in time, polarimetric results on cometary dust contribute to a better understanding of the formation of dust particles, in external regions of the protosolar disk. They likely resulted from submicron-sized grains accreted at low collision velocities, with further addition of minerals processed near the proto-Sun [e.g. 15-16]. At the Late Heavy Bombardment epoch, enormous influxes of interplanetary dust might then have provided a massive delivery of cometary carbonaceous compounds on telluric planets $[1 ; 17]$.

Beyond in space, properties of interplanetary dust may suggest improvements in the modelling of observations of protoplanetary and debris disks. Updating results on the modelling of the dust continuum emission of the protoplanetary disk around MWC 758 [18], with moderately porous spherical particles and the above-mentioned phase functions retrieved by Rosetta, provides a better agreement between synthetic and observed maps of emission at sub-millimetre wavelengths. Besides, observations of debris disks have now shown that light scattering properties of dust in debris disks are far from those predicted by Mie theory and in favour of irregular dust particles, as pointed out essentially by phase functions analysis [19-20]. These results can greatly benefit from in situ and remote cometary observations and from advanced modelling of cometary dust, and at the same time provide examples of dust properties in other stellar systems to put our own Solar System into context [1].

\section{- Conclusions and perspectives}

Polarimetric observations of dust clouds in the Solar System provide clues to the properties of dust particles within cometary comae and the zodiacal cloud. Such results are already noticed to improve the modelling of dust in protoplanetary and debris disks, through the use of irregular porous particles properties instead of more conventional compact spherical ones.

Comet Interceptor, ESA F-Class mission to an Oort-cloud comet, to be launched in 2028, should provide a better understanding of its local polarimetric properties with EnVisS experiment. Meanwhile, remote polarimetric observations of comets might contribute to the target selection.

\section{References}

[1] Levasseur-Regourd, A.C. et al., PSS, 186, 104896, 2020.

[2] Hadamcik, E. et al., PSS, 183, 104527, 2020.

[3] Levasseur-Regourd, A.C. et al.: In Interplanetary dust, E. Grün et al. eds, Springer, 57-94, 2001.

[4] Lasue, J. et al., In Polarimetry of stars and planetary systems, L. Kolokolova et al. eds, CUP, 419-436, 2015.

[5] Lasue et al., PSS, 104973, 2020 (in press).

[6] Levasseur-Regourd et al., PSS, 41, 167-169, 1993.

[7] Fulle, M. et al., AJ, 119, 1968-1977, 2000. 
[8] Lasue, J. et al., Icarus, 199, 129-144, 2009.

[9] Kiselev, N. et al., In Polarimetry of stars and planetary systems, L. Kolokolova et al. eds, CUP, 379-404, 2015.

[10] Muñoz, O. et al., Ap], 247:19, 2020.

[11] Hadamcik, E. et al., MNRAS, 462, S507-S515, 2017.

[12] Rosenbush, V.K., et al., MNRAS, 469, S475-S491, 2017.

[13] Levasseur-Regourd, A.C. et al., Space Sci. Rev., 214:64, 2018.

[14] Levasseur-Regourd, A.C. et al., A\&A, 630, A20, 2019.

[15] Fulle, M. \& Blum, J., MNRAS. 469, S39-S44, 2017.

[16] Lasue, J. et al., A\&A, 630, A28, 2019.

[17] Nesvorný, D., et al., ApJ, 713, 816-836, 2010.

[18] Baruteau, C., et al., MNRAS, 486, 304-319, 2019.

[19] Milli, J., et al., A\&A, 599, A108, 2017.

[20] Milli, J., et al., A\&A, 626, A54, 2019. 